\title{
Gender-specific factors of typical and atypical suicidal behaviors: a secondary data analysis of the 2018 Korea Youth Risk Behavior Survey
}

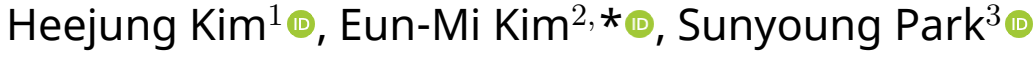 \\ ${ }^{1}$ Mo-Im Kim Nursing Research Institute, College of Nursing, Yonsei University, 03722 Seoul, Republic of Korea \\ ${ }^{2}$ Research Institute of Nursing Science, College of Nursing, Pusan National University, 50612 Yangsan, Republic of Korea \\ ${ }^{3}$ Department of Psychiatry, National Health Insurance Service Ilsan Hospital, 10444 Goyang, Republic of Korea
}

*Correspondence: emkim@pusan.ac.kr (Eun-Mi Kim)

\begin{abstract}
Background and objective: There are limited information about factor associated with linear sequences of suicidal ideation, planning, and attempts among adolescents by gender groups, and those of out-of-linear sequences. The aims of this study was to identify factors associated with typical and atypical patterns of suicidal behaviors by comparing two gender groups of adolescents.

Material and methods: This secondary data analysis was conducted based on the 2018 Korea Youth Risk Behavior Survey $(N=65528)$ by using descriptive statistics and multinomial logistic regression analyses with this complex sample.

Results: A total of $12.4 \%$ of adolescents were in typical groups, and $1.6 \%$ were in atypical groups. Excessive stress and a depressed state were related to typical suicidal behaviors, while violent victimization accounted for atypical suicidal behaviors (all $P$ values $<0.001$ ). In spite of similar degrees and directions in both gender groups, there were some different findings between the gender groups, such as a poor level of academic achievement and stress, as well as living arrangements.

Conclusion: Our study findings provide a comprehensive understanding of the risks of typical and atypical suicidal behaviors in adolescents by considering gender differences. Psychological interventions including school violence prevention should be provided to vulnerable adolescents at risk of suicide, specifically tailored to their gender differences.
\end{abstract}

\section{Keywords}

Adolescent; Depression; Psychological stress; Suicide; Violence

\section{Introduction}

Adolescent suicide is a significant public health concern. South Korea has had the highest suicide rates among countries in the Organization for Economic Cooperation and Development (OECD) since 2011, and adolescents are a major contributing factor in maintaining this high prevalence of suicide, along with elders [1]. The average suicide death rates in South Korean adolescents were 7.6 per 100000 people, a much higher rate than the 5.9 per 100000 people in OECD countries [2]. While suicide rates among other age groups are declining, the suicide rate for adolescents continues to increase, specifically for female adolescents [1]. Thus, there has been an ongoing investigation of how different ages or genders are associated with suicidal behaviors.

Adolescent suicide seems to have reasons different from those of other age groups. First, mental health problems are the leading causes of suicide for adolescents [1, 3], while other causes are reported for other age groups, including financial difficulties for adults and poor health due to disease or functional disabilities for the elderly $[1,2]$. Second, suicide 


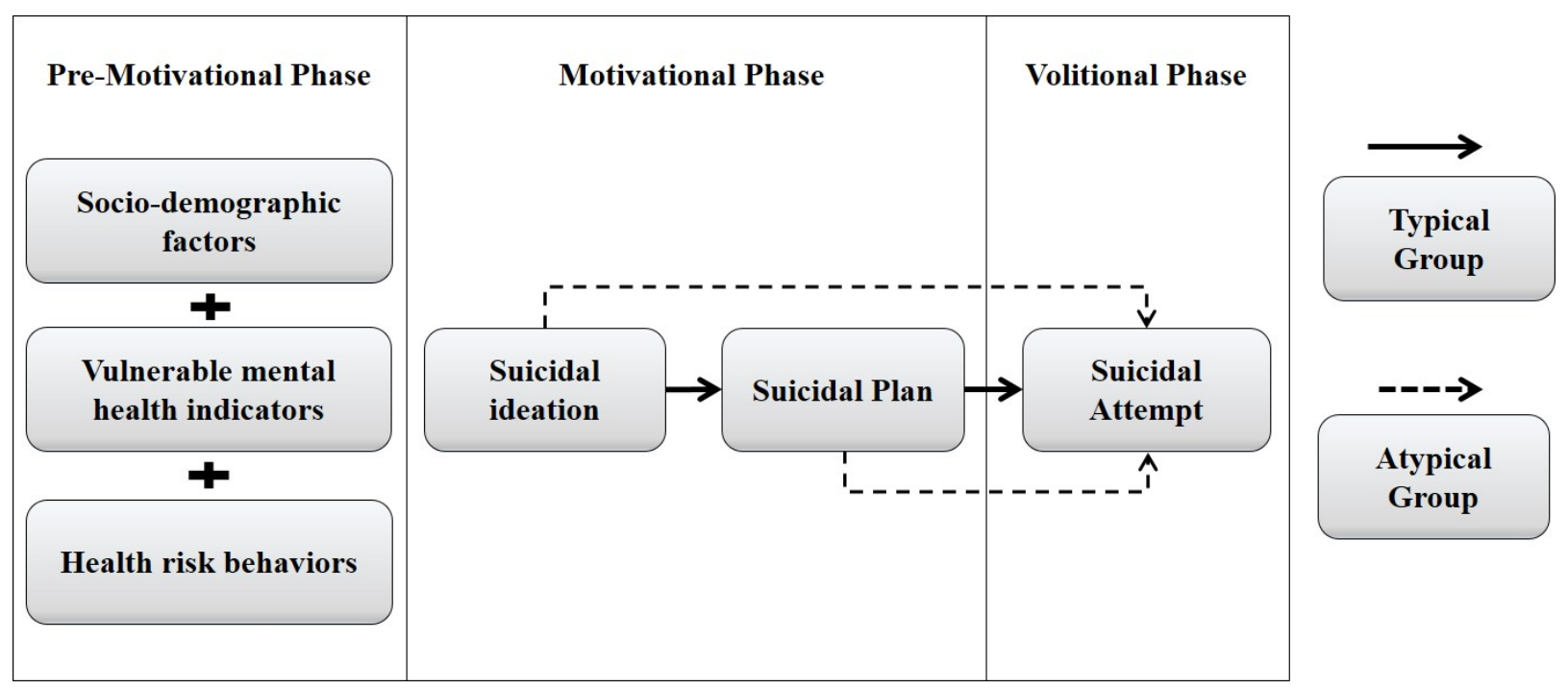

FIG. 1. The conceptual framework of the Integrated Motivational-Volitional model developed by O'Connor (2011) [11].

attempts in adolescents are impulsive or unplanned, while adults and older adults tend to make detailed plans often leading to fatal suicide $[1,2]$. Third, adolescents in South Korea choose different means of suicide, such as jumping, drug misuse, and cutting or stabbing, while adults and older adults choose hanging or drug misuse $[1,2]$. Therefore, it is important to have an in-depth understanding of the suicidal behaviors of adolescents, who are at high risk of uncontrolled suicidal impulses and situational motivations $[1,2]$.

Based on theoretical models explaining suicide, the sequence is often considered on a linear continuum of suicidal ideation, planning, nonfatal attempts, and attempts resulting in death [4, 5]. Most of the suicidal related studies have investigated individuals who reported suicidal ideation [5]. Suicidal ideation is understood as a predisposing factor leading to suicidal attempts [6], but not everyone with suicidal ideation dies by suicide [7]. There are atypical suicides that do not follow the ideation-to-action linear sequence [1, 7]. In other words, some adolescents plan or attempt suicide right away without suicidal ideation $[1,7]$. Recent data has shown that atypical suicide behavior has been frequently reported, specifically in adolescents $[1,7]$. Previous studies have identified psychiatric problems, hopelessness, depression, and impulsivity as factors that could increase the possibility of atypical suicidal progression but do not follow the sequence from suicidal ideation to attempt [4].

However, it is difficult to investigate suicidal planning and attempts owing to the ethical concerns of safety protection for subjects or undue influence by clinicians [5, 8, 9]. Most of the suicidal related studies could have difficulty involving high-risk participants who have planned or attempted suicide actually and who need urgent care or protective agencies $[8,9]$. To overcome contextual, ethical, and methodological challenges, a secondary data analysis with already collected data at the national level would be helpful in identifying the risk factors of typical and atypical suicide behaviors and to develop early detection screening and timely intervention programs for adolescents.

\subsection{Background}

There are several theoretical frameworks to explain the linear sequence of suicidal behaviors from ideation to attempt. First, the ideation-to-action framework proposed by Klonsky and May [10] explained the development and process from suicidal ideation to attempts by focusing on predictors. The ideation-to-action framework differentiates ideations and attempts as separate concepts on suicidal behavior. Second, the integrated motivational-volitional theory explains suicidalconsequent action based on psychological desire [11]. Accumulation and persistence of negative emotions such as defeat and entrapment activate suicidal ideation. Other factors, such as lethal means, planning, and impulsivity, combined with the acquired capability to move forward further the process from suicidal ideation to attempts. Third, the three-step theory of suicide developed by Klonsky and May [10] describes a simplified model for a suicide ideation-to-action framework. While suicidal ideation results from the complexity of psychological pain and hopelessness, suicidal attempts are influenced by dispositional, acquired, and practical factors to increase the capacity to die by suicide. However, these theoretical models have limitations in explaining unexpected behaviors that present atypically as opposed to their proposed linear directions from suicidal ideation to attempt. Thus, there is limited information about the when, how, and why of the transition from suicidal ideation to attempt [4, 5, 10-12].

This study was developed mainly based on the integrated motivational-volitional (IMV) model [11]. The IMV model consists of three phases: (1) the pre-motivational phase describes the biopsychosocial context in which suicidal ideation, plans, and attempts may occur, (2) the motivational phase explains the factors that lead to the emergence of suicidal ideation, and (3) the volitional phase demonstrates the factors that govern the transition from suicidal ideation to suicide attempts and death by suicide. We defined a typical group as those who go through a linear sequence of suicidal ideation, planning, and attempt. In contrast, atypical suicide was defined as those who do not go through a series progressions 
based on this linear sequence. In addition, the variables were selected and categorized based on this framework (see Fig. 1, Ref. [11]). Based on empirical studies, there are diverse types of risk factors related to adolescent suicide behaviors. Family factors include household economic status hardships [13], living situation [13], and familial dynamics or support $[1,14,15]$. School and peer-related factors include academic performance [3] or peer victimization at school [14-17]. Specifically, we focused on factors related to mental health such as high levels of psychological distress and depression [3, $13,15,18,19]$, because mental health problems are the most frequently cited reasons for suicide among Korean youth [1].

There is an ongoing investigation into how different genders associate with adolescent suicide, however, the study findings are inconsistent [1]. Usually, suicidal ideation, planning, and attempts have been more common in female adolescents than male adolescents $[1,13,15,20]$. In a longitudinal study, compared to male adolescents, female adolescents reported suicidal ideation, planning, and attempts more frequently in earlier adolescence [20]. A systemic review with longitudinal studies reported that female adolescents have more attempts, while male adolescents have more deaths by suicide [21]. Gender differences in suicide risk are related to psychological vulnerability, such as depression $[3,18,20$, 21], or different patterns of problem-solving for distress and gender roles in specific cultures [13, 17], which might increase the risk of suicidal behaviors differently. Previous studies examined the risk factor for suicidal ideation in adolescents focusing on the age-by-gender effect [15], and developmental trend of three different suicidal behaviors by gender [20]. For example, compared to male adolescents, female adolescents reported suicidal ideation, planning, and attempts more frequently in earlier adolescence $[15,20]$.

However, previous studies have identified only the risk factors influencing each suicidal ideation, plan or attempt, and reported gender difference in those associations [13-21]. There have been limitations in understanding the typical and atypical pattern of suicidal behaviors, whether they follow the linear sequence from suicidal ideation to attempt by gender. Identifying the features and risk factors of typical or atypical suicidal behaviors among adolescents by gender provides more in-depth information to implement timely suicidal intervention for adolescents. Therefore, we conducted this study to further examine gender differences in adolescent suicide and psychological risk factors, specifically for typical and atypical suicide behaviors among adolescents.

\subsection{Aims}

The aims of this study was to identify how psychologically vulnerable factors relate to suicidal behaviors among adolescents. The specific goals were to (1) estimate the rates of typical and atypical groups of suicidal behaviors; (2) identify associations of psychologically vulnerable factors in typical or atypical groups; and (3) differentiate the significant factors related to typical or atypical groups between the two gender groups.

\section{Methods}

\subsection{Design}

This study was cross-sectional design using secondary data analysis based on data from the 2018 Korea Youth Risk Behavior Survey (KYRBS).

\subsection{Description of primary data and samples}

The KYRBS consisted of nationally representative data collected to investigate the health status of Korean adolescents [22, 23]. The 2018 KYRBS data were collected between April and May 2017. A total of 400 middle schools and 400 high schools were allocated on each stratum using proportional distribution methods. Cluster sampling with twostage stratification was performed randomly, with primary extraction as a school and secondary extraction as a class from strata. In this study a total of 117 strata was considered by 39 local districts (including metropolis, city, town etc.) and three school levels (middle school, general high school, and specialized high school). All students were selected through random sampling based on classified schools and classes $(N=$ 62823). However, the data of total of 60040 students among the participants were selected for analysis after excluding incomplete data or dropout (95.6\% of response rate). The analysis was conducted with the whole sample (see Fig. 2).

\subsection{Data collection}

The KYRBS was conducted after acquiring the selected schools' permission for participation. The survey protocol was explained to the assigned teachers who were involved in the whole process of the survey. A computerized self-report survey was conducted after receiving consent from students who wanted to participate in the study voluntarily. The teacher assigned a single computer to the participating students and placed them in random places. Students were provided with an explanation of the purposes and procedure of the survey. The online system requested that participants answer each question in order to move forward to the next page. The average completion time ranged from 45 to 50 minutes [22].

\subsection{Ethical considerations}

Since 2015, the KYRBS has been conducted without approval from Institutional Review Board (IRB) in the compliance with the Enforcement Regulations of the Bioethics and Safety Act of Korea [22]. The research team explained the purpose and procedure of the KYRBS and obtained informed consent from each student who decided to participate in the survey voluntarily. Any personal identification information was not collected during the survey. This study was reviewed and approved for IRB exemption by the IRB of the researchers' affiliated university (Y-2019-0065). All aspects of the STROBE were considered in this study. 


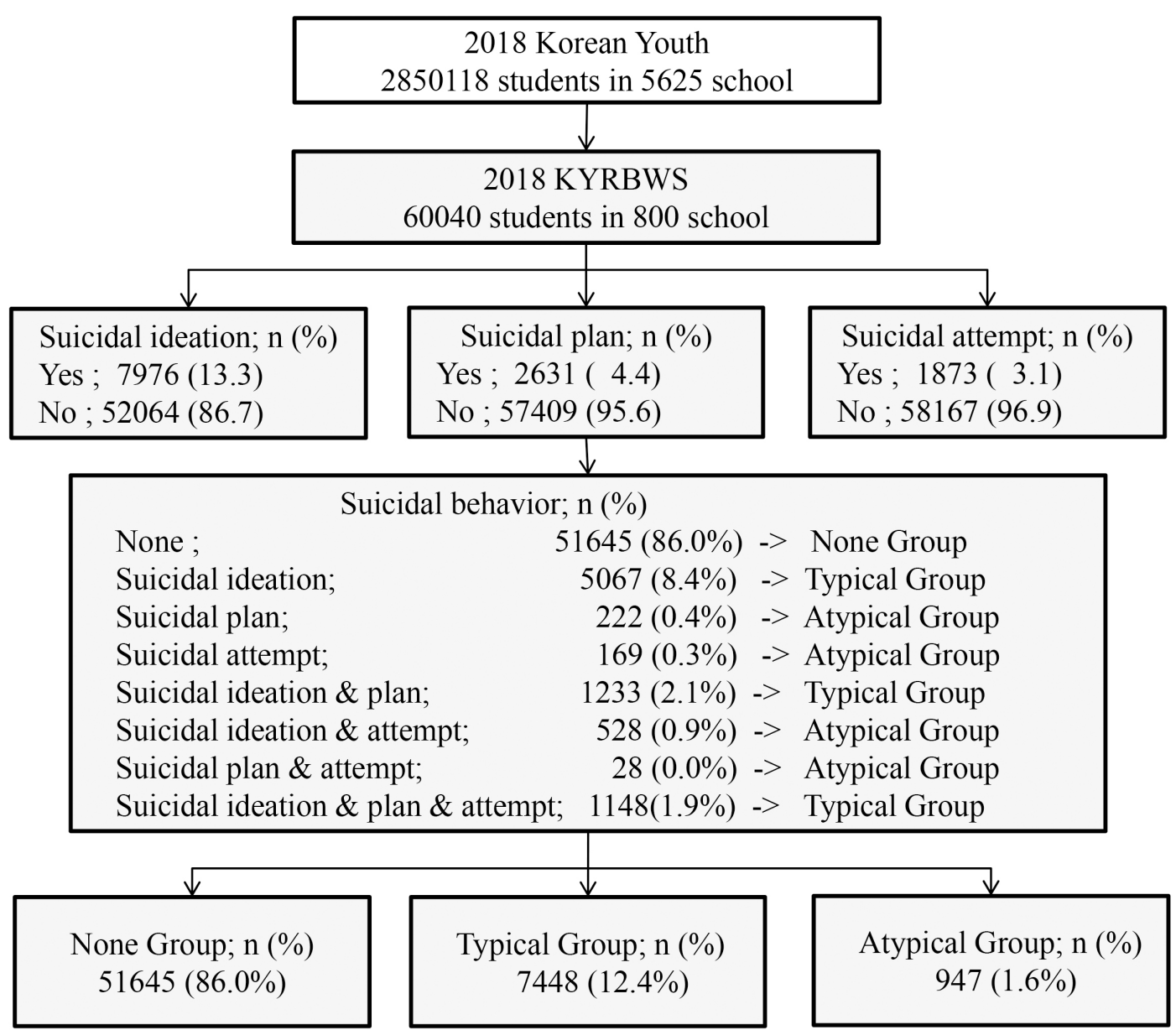

Note: KYRBS: the Korea Youth Risk Behavior Web-based Survey

F IG. 2. Flowchart of study samples.

\subsection{Instrument}

The 2018 KYRBS questionnaire consisted of 15 healthrelated domains, including smoking, drinking, physical activity, mental health, and other factors with 103 items [22]. The variables used in this secondary data analysis are described below.

\subsubsection{Dependent variables}

Three items were selected to operationalize adolescents' suicidal behaviors-suicidal ideation, plans, and attempts. Specific questions included, "Have you had suicidal ideations in the last 12 months?", "Have you had any specific suicidal plans in the last 12 months?", and "Have you made any attempts at suicide in the last 12 months?" The available responses for each question were "yes" and "no", which were coded as 1 or 0 , respectively. The combinations of suicidal behaviors were calculated by summing the total number of responses and grouped as none, typical, and atypical suicide behavioral groups, which were coded as 0,1 or 2, respectively (Fig. 1). A typical group is assumed to follow a linear sequence from suicidal ideation to attempt and categorized adolescents who have (a) suicidal ideation, (b) suicidal ideation and plan, and (c) suicidal ideations, plans, and attempts at the same time
(Fig. 2). The other adolescents had experience with suicide as an atypical group. An atypical group is assumed not to follow a linear sequence from suicidal ideation to attempt and categorized the other patterns of suicidal behaviors; (d) suicidal plan without ideation, (e) suicidal attempt without ideation and plan, (f) suicidal ideation and attempt without plan, and (g) suicidal plan and attempt without ideation.

\subsubsection{Independent variables}

Psychologically vulnerable factors were examined based on stress levels, depressed state, and violent victimization. The specific questions were: (1) "How much stress do you usually feel?", (2) "Have you felt depressed enough in the last 12 months to let it disrupt your daily life for two weeks, and (3) "Have you experienced any serious violence in the last 12 months from a friend, senior, or adult that could have been treated in a hospital (physical assault, intimidation, or bullying, etc.)?" The available responses of stress were "a lot (high)", "to a moderate extent (moderate)", or "don't feel a lot (low)". These were coded as 0,1 , or 2 , respectively. The available responses for depression status and violent victimization were "no" or "yes", which were coded as 0 or 1 , respectively. 


\subsubsection{Control variables}

Socio-demographic factors were self-reported in terms of gender (either "male adolescents" or "female adolescents"), school level ("middle" or "high school"), academic achievement ("excellent", "average", or "poor"), living with family ("yes" or "no"), and household economic status ("affluent", “average", or "poor").

\subsection{Data analysis}

This study followed the KYRBS data analysis guidelines for complex sample data analysis using strata, cluster, finite population correction factors and weight [22]. Listwise deletion was applied to a small amount of missing data (4.4\%). Data analyses were conducted using SPSS version 23.0 (IBM Corp, Chicago, IL, USA) with two-tailed alpha set at 0.05 .

First, descriptive statistics with frequencies and weighted percentages were used to describe socio-demographic variables and different combinations of suicidal behaviors, and three groups of suicidal behaviors ("no", "typical", and "atypical" groups). Second, chi-squared tests based on weighted percentages were used to estimate the rates of typical and atypical groups of suicidal behaviors and differences by sociodemographic and vulnerable mental health factors. Third, multinomial logistic regression analyses with complex samples were used to identify associations of suicidal behaviors and vulnerable mental health factors between the two groups. The reference group in the multinomial logistic regression analyses was the no-suicide behavior group. There was no problem of multicollinearity in conducting the regression analyses; the variance inflation factor of the independent variables ranged from 1.01 to 1.19 and the minimum tolerance was from 0.83 . The results are presented using odds ratios (ORs), $P$-values, and 95\% confidence intervals (CIs).

\section{Results}

\subsection{Characteristics of the sample}

The average age of all sampled adolescents was $15.00 \pm$ 1.77 years, of which $52.1 \%$ were male adolescents and $47.9 \%$ were female adolescents. High school students accounted for $53.6 \%$ of the sample, while middle school students accounted for $46.4 \%$. The academic achievement of adolescents was excellent (38.8\%), average (29.3\%), and poor (31.9\%). The majority of adolescents (95.2\%) lived with their families, and the average or higher household economic status of adolescents was $86.8 \%$. Their stress levels were reported as high (40.4\%), moderate (41.3\%), and low (18.3\%). Among these, $27.1 \%$ reported depression in the past 12 months and $2.1 \%$ received some treatment after experiencing violent victimization (Table 1).

\subsection{Overall rate of typical and atypical groups with suicidal behavior}

Different combinations of suicidal behaviors are presented in Fig. 2. A total of $12.4 \%$ of adolescents were classified in the typical group (4.4\% for male adolescents and $8.0 \%$ for female
T A B L E 1. Descriptive statistics of study variables $(N=$ 60040).

\begin{tabular}{|c|c|c|}
\hline Variable & Categories & $\mathrm{n}(\%)^{a}$ \\
\hline \multicolumn{3}{|l|}{ Socio-demographic factors } \\
\hline \multirow{2}{*}{ Gender } & Male & $30463(52.1)$ \\
\hline & Female & $29577(47.9)$ \\
\hline \multirow{2}{*}{ School level } & Middle school & $30228(46.4)$ \\
\hline & High school & $29812(53.6)$ \\
\hline \multirow{3}{*}{ Academic achievement } & Excellent & $23420(38.8)$ \\
\hline & Average & $17526(29.3)$ \\
\hline & Poor & $19094(31.9)$ \\
\hline \multirow{2}{*}{ Living with family } & Yes & $56654(95.2)$ \\
\hline & No & $3386(4.8)$ \\
\hline \multirow{3}{*}{ Households economic status } & Affluent & $24207(40.8)$ \\
\hline & Average & $27808(46.0)$ \\
\hline & Poor & $8025(13.2)$ \\
\hline \multicolumn{3}{|l|}{ Poor mental health indicators } \\
\hline \multirow{3}{*}{ Stress $^{b}$} & High & $24312(40.4)$ \\
\hline & Middle & $24638(41.3)$ \\
\hline & Low & $11090(18.3)$ \\
\hline \multirow{2}{*}{ Depression $^{b}$} & Yes & $16208(27.1)$ \\
\hline & No & $43832(72.9)$ \\
\hline \multirow{2}{*}{ Violent victimization } & Yes & $1242(2.1)$ \\
\hline & No & $58798(97.9)$ \\
\hline
\end{tabular}

Note: ${ }^{a}$ Percentage with complex sample was conducted. ${ }^{b}$ In the last 12 months.

adolescents, respectively) and $1.6 \%$ were in the atypical group ( $0.6 \%$ for male adolescents and $1.0 \%$ for female adolescents, respectively).

\subsection{Psychologically vulnerable factors related to typical and atypical suicidal behaviors}

The results of the multinomial logistic regression analyses are shown in Table 2. The Cox and Snell $\mathrm{R}^{2}$ was 0.202 , and the Nagelkerke $\mathrm{R}^{2}$ was 0.339 , showing that the independent variables explained the classification of typical and atypical groups compared to the no suicide behavioral group [24]. All independent variables were associated statistically with suicidal behaviors (all $P$-values of Omnibus Wald F-tests < $0.001)$.

Of the socio-demographic factors, the factors that increased the likelihood of experiencing suicidal behavior, for either typical or atypical groups, were female adolescents who were studying in middle school rather than high school, had poor academic achievement, lived apart from their families, and had poor household economic status (all $P$-values $<0.001)$. In general, the ORs of these identified factors were much higher in the atypical group than in the typical group. Specifically, ORs of either the typical or atypical group increased when they had much poorer academic achievement and household economic status.

Psychological vulnerability factors increased the likelihood of experiencing suicidal behavior, either for typical or atypical groups, when they reported moderate or high levels of stress, depressed states within 12 months, and past experience of violent victimization (all $P$-values $<0.001$ ). However, the 
T A B L E 2. Multivariate Multinomial Logistic Regression of Suicidal Behavior Group $(N=60040)$.

\begin{tabular}{|c|c|c|c|c|}
\hline \multirow{2}{*}{ Variable } & \multirow{2}{*}{ Categories } & \multicolumn{2}{|c|}{$\operatorname{AOR}[95 \% \mathrm{CI}]^{a}$} & \multirow{2}{*}{ Wald F } \\
\hline & & Typical group & Atypical group & \\
\hline \multicolumn{5}{|l|}{ Socio-demographic factors } \\
\hline Gender (ref. Male) & Female & $1.31[1.23-1.40]$ & $1.43[1.24-1.64]$ & $44.13^{* * *}$ \\
\hline School level (ref. High school) & Middle school & $1.36[1.27-1.45]$ & $1.81[1.55-2.12]$ & $64.25^{* * *}$ \\
\hline \multirow{2}{*}{ Academic achievement (ref. Excellent) } & Average & $0.95[0.89-1.03]$ & $1.20[1.02-1.45]$ & $11.38^{* * *}$ \\
\hline & Poor & $1.13[1.05-1.21]$ & $1.48[1.27-1.74]$ & \\
\hline Living with family (ref. Yes) & No & $1.30[1.15-1.46]$ & $1.57[1.23-2.01]$ & $15.63^{* * *}$ \\
\hline \multirow{2}{*}{ Households economic status (ref. Affluent) } & Average & $0.98[0.93-1.05]$ & $1.00[0.85-1.17]$ & $32.02^{* * *}$ \\
\hline & Poor & $1.44[1.33-1.55]$ & $1.60[1.33-1.92]$ & \\
\hline \multicolumn{5}{|l|}{ Poor mental health indicators } \\
\hline \multirow{2}{*}{$\operatorname{Stress}^{b}$ (ref. Low) } & High & $5.54[4.09-6.27]$ & $2.41[1.87-3.09]$ & $388.89^{* * *}$ \\
\hline & Middle & $1.64[1.44-1.87]$ & $1.04[0.80-1.35]$ & \\
\hline Depression $^{b}$ (ref. No) & Yes & $9.03[8.47-9.63]$ & $5.72[4.93-6.63]$ & $2450.41^{* * *}$ \\
\hline Violent victimization (ref. No) & Yes & $3.96[3.40-4.60]$ & $7.42[5.81-9.46]$ & $220.14^{* * *}$ \\
\hline Cox \& Snell R ${ }^{2}$ & \multicolumn{3}{|c|}{0.202} & \\
\hline Nagelkerke $\mathrm{R}^{2}$ & \multicolumn{3}{|c|}{0.339} & \\
\hline
\end{tabular}

T A B L E 3. Multivariate Multinomial Logistic Regression of Suicidal Behavior Group by Gender $(N=60040)$.

\begin{tabular}{|c|c|c|c|c|c|c|c|}
\hline \multirow{3}{*}{ Variables } & \multirow{3}{*}{ Categories } & \multicolumn{3}{|c|}{ Male } & \multicolumn{3}{|c|}{ Female } \\
\hline & & \multicolumn{2}{|c|}{$\operatorname{AOR}[95 \% \mathrm{CI}]^{a}$} & \multirow{2}{*}{ Wald F } & \multicolumn{2}{|c|}{$\operatorname{AOR}[95 \% \mathrm{CI}]^{a}$} & \multirow{2}{*}{ Wald F } \\
\hline & & Typical group & Atypical group & & Typical group & Atypical group & \\
\hline \multicolumn{8}{|l|}{ Socio-demographic factors } \\
\hline School level (ref. High school) & Middle school & $1.20[1.08-1.33]$ & $1.62[1.28-2.06]$ & $13.52^{* * *}$ & $1.48[1.36-1.61]$ & $1.99[1.64-2.40]$ & $59.76^{* * *}$ \\
\hline \multirow{2}{*}{ Academic achievement (ref. Excellent) } & Average & $1.01[0.89-1.14]$ & $1.37[1.04-1.81]$ & \multirow{2}{*}{$2.42^{*}$} & $0.92[0.84-1.01]$ & $1.12[0.87-1.44]$ & \multirow{2}{*}{$10.00^{* * *}$} \\
\hline & Poor & $1.11[0.99-1.25]$ & $1.35[1.03-1.76]$ & & $1.14[1.05-1.23]$ & $1.58[1.27-1.96]$ & \\
\hline Living with family (ref. Yes) & No & $1.43[1.19-1.72]$ & $1.38[0.94-2.03]$ & $8.36^{* * *}$ & $1.19[1.03-1.37]$ & $1.74[1.24-2.45]$ & $7.68^{* * *}$ \\
\hline \multirow{2}{*}{ Households economic status (ref. Affluent) } & Average & $0.92[0.83-1.02]$ & $0.84[0.65-1.09]$ & \multirow{2}{*}{$8.59^{* * *}$} & $1.03[0.95-1.11]$ & $1.14[0.92-1.39]$ & \multirow{2}{*}{$24.55^{* * *}$} \\
\hline & Poor & $1.33[1.16-1.52]$ & $1.42[1.05-1.91]$ & & $1.52[1.38-1.68]$ & $1.77[1.38-2.26]$ & \\
\hline \multicolumn{8}{|l|}{ Poor mental health indicators } \\
\hline \multirow{2}{*}{ Stress $^{b}$ (ref. Low) } & High & $5.05[4.30-5.94]$ & $1.66[1.20-2.29]$ & \multirow{2}{*}{$173.17^{* * *}$} & $6.25[5.03-7.76]$ & $4.44[2.82-7.00]$ & \multirow{2}{*}{$214.87^{* * *}$} \\
\hline & Middle & $1.44[1.20-1.72]$ & $0.85[0.63-1.16]$ & & $1.91[1.54-2.37]$ & $1.70[1.05-2.75]$ & \\
\hline Depression $^{b}$ (ref. No) & Yes & $10.75[9.69-11.75]$ & $6.47[5.07-8.26]$ & $1144.54^{* * *}$ & $7.96[7.35-8.62]$ & $5.18[4.30-6.25]$ & $1428.67^{* * *}$ \\
\hline Violent victimization (ref. No) & Yes & $3.72[3.05-4.53]$ & $7.74[5.70-10.53]$ & $138.58^{* * *}$ & $4.26[3.34-5.44]$ & $7.14[4.93-10.35]$ & $87.74^{* * *}$ \\
\hline Cox \& Snell $\mathrm{R}^{2}$ & & 0.175 & & & 0.212 & & \\
\hline Nagelkerke $\mathrm{R}^{2}$ & & 0.336 & & & 0.323 & & \\
\hline
\end{tabular}

Note: Regression reference category: no suicidal behavior group.

${ }^{a}$ Logistic regression with complex sample was conducted. ${ }^{b}$ In the last 12 months. ${ }^{*} P<0.05$. ${ }^{* * *} P<0.001$.

AOR, Adjusted odds ratio; ref, reference of each independent variable group.

ORs of the typical and atypical groups were somewhat different. Adolescents with high stress are more likely to be involved in the typical group with 5.54 times (95\% CI: 4.096.27 ), and the atypical group with 2.41 times (95\% CI: $1.87-$ 3.09), than adolescents with low stress. Adolescents with depression are more likely to be involved in the typical group with 9.03 times (95\% CI: 8.47-9.63) and the atypical group with 5.72 times (95\% CI: 4.93-6.63). Adolescents who have experienced violence are more likely to be involved in the typical group with 3.96 times (95\% CI: 3.40-4.60) and the atypical group with 7.42 times (95\% CI: 5.81-9.46).

\subsection{Gender differences in associated factors between typical and atypical suicide-behavioral groups}

Most of the significant variables identified in Table 3 showed similar degrees and directions in both gender groups. In terms of socio-demographics, the likelihood of experiencing suicidal behavior, specifically for the atypical group, increased when they studied in middle school more than in high school and had poor household economic status (all $P$-values $<0.001)$. In addition, psychological vulnerability factors increased the likelihood of experiencing suicidal behavior, specifically for the typical group, when they reported high 
or moderate levels of stress and depression status within 12 months as well as the past experience of violent victimization for the atypical group (all $P$-values $<0.001$ ).

However, the ORs of the typical and atypical groups were somewhat different by gender group. Male adolescents with high stress are more likely to involved in the typical group with 5.05 times (95\% CI: 4.30-5.94), and the atypical group with 1.66 times (95\% CI: 1.20-2.29), than adolescents with low stress. Female adolescents with high stress are more likely to be involved in the typical group with 6.25 times (95\% CI: 5.03-7.76) and the atypical group with 4.44 times (95\% CI: 2.82-7.00). Male adolescents with depression are more likely to be involved in the typical group with 10.75 times (95\% CI: 9.69-11.75) and the atypical group with 6.47 times (95\% CI: 5.07-8.26). Female adolescents with depression are more likely to be involved in the typical group with 7.96 times (95\% CI: 7.35-8.62), and the atypical group with 5.18 times (95\% CI: 4.30-6.25). Male adolescents with the experience of violence are more likely to be involved in the typical group with 3.72 times (95\% CI: 3.05-4.53), and the atypical group with 7.74 times (95\% CI: 5.70-10.53). Female adolescents with the experience of violence are more likely to be involved in the typical group with 4.26 times (95\% CI: 3.34-5.44), and in the atypical group with 7.14 times (95\% CI: 4.93-10.35).

\section{Discussion}

Our study explores the rates of suicidal behaviors by classifying them into typical and atypical patterns and examines the gender difference in the associated factors, specifically focusing on psychological vulnerability factors. To our knowledge, this study is the first attempt to identify gender differences in the associations between psychological vulnerability factors and suicidal behaviors using nationally representative data. Excessive stress, a depressed state, and violent victimization were associated with typical or atypical suicide-behaviors in both gender groups. The ORs, which indicated the relative strength of the association between psychologically vulnerable factors and typical or atypical suicidal behaviors, differed by gender group. The findings of this study, which provide more in-depth information to understand the suicidal behaviors of adolescents, could be used as evidence for suicidal prevention programs for adolescents in schools and communities.

Overall, adolescent suicidal behaviors are more prevalent in the typical pattern than in the atypical pattern in both gender groups. Adolescent suicidal ideation could lead to suicidal plans and attempts based on the established backgrounds of relevant theories [4, 10]. This predisposing tendency may be shown in the national statistics-that is, the rate of suicidal ideation among adolescents was much higher than that of suicidal plans and attempts $[23,25]$. The significant risk factors reported for suicide, such as psychiatric disorders, hopelessness, depression, and impulsivity were more strongly related not only to typical suicidal progression from suicidal ideation to attempts but also atypical suicidal behaviors [4]. However, many studies emphasize that we might overlook adolescents who directly attempt fatal suicide without a linear sequence of suicidal ideation to suicidal attempt [1]. Recently, it has become important to understand that we may encounter unexpected suicide behaviors among highly predictable victims $[7,26]$; thus, it is important to identify those who might harm themselves without suicide ideation.

In this study, the ORs of socio-demographic factors that are expected to be related with high suicidality were much higher in the atypical group than those in the typical group, thus the information would be helpful for this early identification of the diverse type of pattern $[7,9]$. The higher risk of atypical suicide behaviors was found in female adolescents, middle school students, students with poor academic achievement, low income, and those who had experienced violent victimization. Younger adolescents with low levels of academic performance and incomes were more likely to have higher levels of impulsivity regarding suicidal behaviors $[13,20]$. In addition, female adolescents were more likely to engage in both typical and atypical suicidal behaviors compared to male adolescents, but gender discrepancies increased in the atypical group more than that of the typical group. Previous studies recognized that female adolescents have more frequent suicidal behaviors in earlier adolescence than male adolescents in terms of ideation, planning, and attempts $[13,20]$. It might be relevant that female adolescents have a greater risk of internalized disorders (e.g., depression or other mood disorders) [21]. Thus, these factors should be included in early assessment tools to identify the high-risk groups for atypical suicide in a specific gender group.

Our findings confirm the importance of improving mental health for suicide prevention. The findings of this study showed that adolescents with high stress are more likely to be involved in the typical and atypical group at 5.54 times (5.05 times in male vs 6.25 time in female) and 2.41 times (1.66 times in male vs 4.44 time in female), respectively. Adolescents with depression are more likely to be involved in the typical and atypical group with 9.03 (10.75 times in male vs 7.96 time in female) and 5.72 times (6.47 times in male vs 5.18 times in female), respectively. Higher levels of stress and recently depressed states were more likely to lead to suicidal behaviors, which is more significant in the typical group than in the atypical group. Consistent with previous studies, more suicidal behaviors in adolescents were related to higher levels of psychological distress $[3,13,19,27]$ and depression $[13,18]$. The life stress and depression of adolescents results from the high expectations of academic achievement, college entrance examinations, and poor interpersonal relationships with parents and peers $[3,15,19]$. Thus, it is required to reduce the negative impact of uncontrolled stress and depression for the vulnerable adolescent at high risk of suicidal behaviors $[15,18]$. Specifically, psychological distress related to life events may trigger suicidal ideation to move forward to planning and attempts when stress accumulates excessively [27]. In addition, our findings support the risk of suicidal behaviors related to depression [3, 13, 18], specifically for the atypical pattern. This finding is critically important for monitoring the level of adolescents' stress and depression in 
a timely manner $[15,18]$.

In addition, the results of this study provide more detailed information that female adolescents with high stress were more likely to be involved in the atypical group, while male adolescents with depression were more likely to involved in the typical group. There was a limitation in comparing the findings of this study as little previous research has been done on typical and atypical suicidal behaviors. Systematic and sustainable investigation by gender of trajectories of suicidal ideation, plans, and attempts will provide an accurate picture of developmental patterns of suicidal episodes among adolescents $[15,20]$.

Adolescents who have experienced violence are more likely to be involved in the typical and atypical group with 3.96 (3.72 times in male vs 7.74 times in female) and 7.42 times (4.26 times in male vs 7.14 times in female), respectively. Interestingly, these results mean that adolescents who have experienced violent victimization were more likely to belong to the typical and atypical group when compared to adolescents who have not experienced violent victimization. These results are consistent with findings that bullying is related to increased risk for suicidal behavior not only typical and atypical patterns [14-17, 28]. A meta-analysis found that many types of bullying, such as bullying victimization, bullying perpetration, and bullyvictim status show significant associations with a higher likelihood of suicidal behavior [17].

Notably, this study revealed that bullied adolescents are more likely to have atypical suicide behaviors without sequential processes from ideations to plans and attempts with more increased OR. In addition, the results of this study demonstrated that female adolescents who had experienced violent victimization were more likely to be involved in the atypical group. It was difficult to compare the results of this study because there has been little previous study on associations of atypical suicidal behaviors or gender differences. It could be explained that bullied adolescents tend to externalize or internalize their difficulties or personal problems $[21,28]$. A Chinese study found that suicide attempters without reported ideation were likely to be more impulsive, had the intent to hurt others, sought less support from their significant others, and consulted psychiatric doctors for medical help [26]. Thus, the aggression experienced in being bullied could target others and themselves, representing self-harm or suicidal behaviors [28]. Moreover, the victims were isolated from school to keep them distant from the perpetrator, making the victim disconnected from social capital and deprived of coping opportunities [16]. Bullied adolescents might have difficulty asking for help and having close interpersonal relationships; therefore, one important role of school nurses is to screen out adolescents who are being bullied with insightful attention and provide supportive programs to adolescents to show them how bullying is associated with psychological harm $[15,21]$. It would be helpful to provide school-based mental health screening tests periodically and build close partnerships with teachers, parents, and peers to prevent adolescent violence [21, 28].
Integrative programs containing both adolescent suicide and the prevention of victimization should be developed and tested to ensure the synergetic effects of better adolescent safety [16]. It would also be effective to develop a differentiated strategy that considers gender in providing such intervention $[15,20]$.

Adolescents not living with their families could have an increased likelihood of both typical and atypical suicidal behaviors. Living with family not only means a stable environment for living, but also refers to a support system providing interpersonal relationships and utilizable resources [21]. Based on Durkheim's [29] social integration theory of suicide, family is the key component to form the social capital of adolescents that functions as a buffering system between suicide and poor mental health [13, 19]. Thus, adolescents who do not live with their families may have difficulty using their families as a buffer. Based on the three-step theory [10], connectedness could function as a protective factor against suicide in general. Family cohesion and connectedness may protect adolescents from self-harmful behaviors [3]. Particularly, our study revealed gender differences in association between living with family and suicidal behaviors; male adolescents who do not live with their families showed typical suicidal behaviors, while female adolescents who do not live with their families often showed atypical suicidal behavior patterns. Thus, it would be necessary to strengthen other social support systems that replace the family system by considering gender differences in patterns of suicidal behavior.

\section{Study limitations and future research}

There are some limitations to this study. First, our study was cross-sectional in design; thus it is difficult to infer causality in our study findings. Second, due to self-reporting, adolescent suicide might be under-reported because of social stigma or associated emotional distress [8]. Third, stress levels, depression status, and violent victimization were measured using a single item each, so using valid measures with multiple questions will provide in-depth understanding. Fourth, suicidal ideation was measured using a single item, so it is limited to assess passive (wishing to be gone) versus active (planning means) suicidal behavior that could more fully describe typical versus atypical groups. Fifth, without suicidal ideation, suicide planning and attempts are unusual cases; this study is limited in the profound understanding of these adolescents. Thus, we suggest future research using more sophisticated designs and objective measurement for clinical diagnosis and practice $[3,18]$. Longitudinal studies including a large number of adolescents could identify other considerable factors associated with suicidal behaviors, and qualitative studies based on in-depth personal interviews with typical and atypical suicide-behavioral groups across the developmental stage and more accumulated data about the transition from plan to attempt. 


\section{Conclusions}

Previous studies of suicide have primarily investigated only suicidal ideation and typical pattern in linear sequence. Our study found that a considerable number of adolescents engage in both typical and atypical suicidal behaviors based on nationally representative data. Our findings support significant associations of psychological vulnerability factors with suicidal behaviors that are somewhat different for each gender. Moreover, our findings support the importance of managing adolescent mental health problems to prevent both typical and atypical patterns of suicidal behaviors. Psychological interventions and the prevention of school violence should be undertaken for vulnerable adolescents at risk of suicide. In addition, the development of gender-specific strategies is required to overcome specific risk factors of suicide. Thus, cooperation will be necessary between school, family, and students themselves.

\section{Implications}

Our findings provide comprehensive evidence for mental health nurses and policymakers, who need to reduce suicidal behaviors among adolescents with psychologically vulnerable health. National policies against suicide need to be refined based on recent trends in adolescent culture and behavioral changes [1]. In general, it would be helpful to conduct relevant gatekeeper training for teachers, family, community leaders, and others to aid early detection, improve rapid response, and support referral for hospital treatment for adolescents at high risk of suicide $[1,30,31]$. It is necessary to use the comprehensive assessment tool of suicide risk in a school setting [32]. Specifically, family might be a key collaborator in providing proper interpersonal relationships and communication [31], especially for female adolescents with atypical suicidal behaviors [13]. At the same time, it is important to protect the confidentiality and autonomy of high-risk adolescents in terms of information sharing and referral to clinicians [8].

Gender-specific programs are very important in preventing suicidal behaviors at school $[13,33]$. Stress management, depression treatment, and anti-victimization should be key components when designing suicidal prevention programs in middle and high school for typical suicidal behaviors [1,33]. The most vulnerable subgroups with suicidal behaviors and psychologically vulnerable health should be screened using valid instruments-for example, the adolescent health and problem behavior screening questionnaire-II [15]. Mental health nurses and school nurses are in an important position to help develop school-based suicidal prevention programs making use of an effective method for educating adolescents in recognizing their own mental health conditions and enhancing adolescent help-seeking abilities [14, 34-36]. There are significant gender differences in psychological factors with suicidal behaviors, thus it would be important to take gender difference into account with any program development [20]. Usually, male adolescents deal with depressive symptoms with deviance or health risks behaviors, such as alcohol or cigarettes, so that their psychological problems are often overlooked [37], while female adolescents ask for help from people around them and seek early treatment more readily through alliances for their psychological problems, such as getting advice in family systems. Thus, genderspecific strategies should be developed to increase change in suicidal behavior against poor mental health.

\section{Author contributions}

HK and EMK contributed to the conception and/or design of the study and analyzed the data. SYP provide extensive contribution with clinical expertise on the topic. All authors participated in interpreting the results and writing the manuscript. All authors involved in developing this manuscript to meet the authorship criteria and agree with the final version of this manuscript.

\section{Ethics approval and consent to participate}

This study conducted secondary data analysis using the 2018 Korea Youth Risk Behavior Web-based Survey. The Institutional Review Board exemption from the research's affiliated University was obtained prior to data analyses (approval no.: 2017-2445-001).

\section{Acknowledgment}

The researchers appreciate Korean Ministry of Education, Korean Ministry of Health and Welfare \& Korean Centers for Disease Control and Prevention for data sharing and advising for the analyses in this secondary data analysis. The researchers also thank to all respondents in this survey for their time and sharing their experience.

\section{Funding}

This study was supported by the Ministry of Science, ICT and Future Planning (NRF-2020R1C1C1012848).

\section{Conflict of interest}

The authors declare no conflict of interest.

\section{References}

[1] Korean Ministry of Health and Welfare and Korea Suicide Prevention Center. 2020 Suicide Prevention White Book. Seoul, Korea: Korea Suicide Prevention Center. 2020.

[2] Korean Ministry of Health and Welfare and Korea Suicide Prevention Center. 2018 Suicide Prevention White Book. Seoul, Korea: Korea Suicide Prevention Center. 2018.

[3] Arun P, Garg R, Chavan BS. Stress and suicidal ideation among adolescents having academic difficulty. Industrial Psychiatry Journal. 2017; 26: 64-70

[4] Klonsky ED, May AM. Differentiating suicide attempters from suicide ideators: a critical frontier for suicidology research. Suicide \& LifeThreatening Behavior. 2014; 44: 1-5.

[5] Klonsky ED, May AM, Saffer BY. Suicide, Suicide Attempts, and Suicidal Ideation. Annual Review of Clinical Psychology. 2016; 12: $307-330$. 
[6] Oppong Asante K, Kugbey N, Osafo J, Quarshie EN, Sarfo JO. The prevalence and correlates of suicidal behaviours (ideation, plan and attempt) among adolescents in senior high schools in Ghana. SSM Population Health. 2017; 3: 427-434.

[7] Harmer B, Lee S, Duong TVH, Saadabadi A. Suicidal ideation. 2021. In: StatPearls. Treasure Island (FL): StatPearls Publishing. 2020.

[8] Lakeman R, Fitzgerald M. Ethical suicide research: a survey of researchers. International Journal of Mental Health Nursing. 2009; 18: $10-17$.

[9] National Institute of Mental Health. Conducting research with participants at elevated risk for suicide: considerations for researchers. 2020. Available at: https://www.nimh.nih.gov/ funding/clinical-research/conducting-researchwith-participants-at-elevated-risk-for-suicideconsiderations-for-researchers.shtml (Assessed: 15 December 2020).

[10] Klonsky ED, May AM. The Three-Step Theory (3ST): a New Theory of Suicide Rooted in the "Ideation-to-Action" Framework. International Journal of Cognitive Therapy. 2015; 8: 114-129.

[11] O'Connor RC. Towards an integrated motivational-volitional model of suicidal behaviour. In: O'Connor RC, Platt S, Gordon J, eds. International handbook of suicide prevention: Research, policy and practice (pp. 181-198). Chichester, UK: Wiley. 2011.

[12] O'Carroll PW, Berman AL, Maris RW, Moscicki EK, Tanney BL, Silverman MM. Beyond the Tower of Babel: a nomenclature for suicidology. Suicide \& Life-Threatening Behavior. 1996; 26: 237-252.

[13] Lee G, Choi Y. Association of school, family, and mental health characteristics with suicidal ideation among Korean adolescents. Research in Nursing \& Health. 2015; 38: 301-310.

[14] Doyle L, Treacy MP, Sheridan A. Self-harm in young people: Prevalence, associated factors, and help-seeking in school-going adolescents. International Journal of Mental Health Nursing. 2015; 24: 485-494.

[15] Lee D, Jung S, Park S, Hong HJ. The impact of psychological problems and adverse life events on suicidal ideation among adolescents using nationwide data of a school-based mental health screening test in Korea. European Child \& Adolescent Psychiatry. 2018; 27: 1361-1372.

[16] Bauman S, Toomey RB, Walker JL. Associations among bullying, cyberbullying, and suicide in high school students. Journal of Adolescence. 2013 ; 36: 341-350.

[17] Holt MK, Vivolo-Kantor AM, Polanin JR, Holland KM, DeGue S, Matjasko JL, et al. Bullying and suicidal ideation and behaviors: a metaanalysis. Pediatrics. 2015; 135: e496-e509.

[18] Avenevoli S, Swendsen J, He J, Burstein M, Merikangas KR. Major depression in the national comorbidity survey-adolescent supplement: prevalence, correlates, and treatment. Journal of the American Academy of Child and Adolescent Psychiatry. 2015; 54: 37-44.

[19] Bae SM. Individual and social stress, social capital, and depressive symptoms as predictors of adolescent suicide in South Korea: a mediated moderation model. Journal of Health Psychology. 2019; 24: 1909-1919.

[20] Boeninger DK, Masyn KE, Feldman BJ, Conger RD. Sex differences in developmental trends of suicide ideation, plans, and attempts among European American adolescents. Suicide \& Life-Threatening Behavior. 2010; 40: 451-464.

[21] Miranda-Mendizabal A, Castellví P, Parés-Badell O, Alayo I, Almenara J, Alonso I, et al. Gender differences in suicidal behavior in adolescents and young adults: systematic review and meta-analysis of longitudinal studies. International Journal of Public Health. 2019; 64: 265-283.

[22] Korean Ministry of Education, Korean Ministry of Health and Welfare \& Korean Centers for Disease Control and Prevention. Guidelines for the Use of the 14th Korea Youth Risk Behavior Web-based Survey. 2018. Available at: https : / www . kdca.go.kr/yhs/ (Accessed: 20 June 2019).

[23] Korean Ministry of Education, Korean Ministry of Health and Welfare \& Korea Centers for Disease Control and Prevention. The Fourteenth Korea Youth Risk Behavior Web-based Survey Statistics. 2018. Available at: https : / www.kdca.go.kr/yhs/ (Accessed: 20 June 2019).

[24] Smith TJ, McKenna CM. A comparison of logistic regression pseudo $\mathrm{R}^{2}$ indices. Multiple Linear Regression Viewpoints. 2013; 39: 17-26.

[25] Centers for Disease Control and Prevention. Youth risk behavior survey data summary \& trends reports 2007-2017. 2018. Available at: https://www.cdc.gov/healthyyouth/data/yrbs/pdf/ trendsreport.pdf (Assessed: 1 March 2021).

[26] Wei S, Li H, Hou J, Chen W, Tan S, Chen X, et al. Comparing characteristics of suicide attempters with suicidal ideation and those without suicidal ideation treated in the emergency departments of general hospitals in China. Psychiatry Research. 2018; 262: 78-83.

[27] Liu RT, Miller I. Life events and suicidal ideation and behavior: a systematic review. Clinical Psychology Review. 2014; 34: 181-192.

[28] Kelly EV, Newton NC, Stapinski LA, Slade T, Barrett EL, Conrod PJ, et al. Suicidality, internalizing problems and externalizing problems among adolescent bullies, victims and bully-victims. Preventive Medicine. 2015; 73: 100-105.

[29] Durkheim E. Suicide: A Study in Sociology. 2nd edn. New York: The Free Press. 1951

[30] McAndrew S, Warne T. Hearing the voices of young people who self-harm: implications for service providers. International Journal of Mental Health Nursing. 2014; 23: 570-579.

[31] Manuel J, Crowe M, Inder M, Henaghan M. Suicide prevention in mental health services: a qualitative analysis of coroners' reports. International Journal of Mental Health Nursing. 2018; 27: 642-651.

[32] Diamond GS, Herres JL, Krauthamer Ewing ES, Atte TO, Scott SW, Wintersteen MB, et al. Comprehensive Screening for Suicide Risk in Primary Care. American Journal of Preventive Medicine. 2017; 53: 4854.

[33] Miller AB, Eisenlohr-Moul T, Giletta M, Hastings PD, Rudolph KD, Nock MK, et al. A within-person approach to risk for suicidal ideation and suicidal behavior: Examining the roles of depression, stress, and abuse exposure. Journal of Consulting and Clinical Psychology. 2017; 85: 712-722.

[34] King KA, Strunk CM, Sorter MT. Preliminary effectiveness of surviving the teens $\left({ }^{\circledR}\right)$ suicide prevention and depression awareness program on adolescents' suicidality and self-efficacy in performing help-seeking behaviors. Journal of School Health. 2011; 81: 581-590.

[35] McCabe R, Garside R, Backhouse A, Xanthopoulou P. Effectiveness of brief psychological interventions for suicidal presentations: a systematic review. BMC Psychiatry. 2018; 18: 120.

[36] Wasserman D, Hoven CW, Wasserman C, Wall M, Eisenberg R, Hadlaczky G, et al. School-based suicide prevention programmes: the SEYLE cluster-randomised, controlled trial. Lancet. 2015; 385: 15361544.

[37] Naninck EFG, Lucassen PJ, Bakker J. Sex Differences in Adolescent Depression: do Sex Hormones Determine Vulnerability? Journal of Neuroendocrinology. 2011; 23: 383-392. 